EM

\title{
FRONTERA E INTEGRACIÓN REGIONAL EN SALUD: ACCESO A LOS SISTEMAS DE SALUD DE POSADAS - ENCARNACIÓN
}

\author{
INTEGRAÇÃO FRONTEIRIÇA E REGIONAL EM SAÚDE: ACESSO AOS \\ SISTEMAS DE SAÚDE DE POSADAS - ENCARNAÇÃO ${ }^{1}$
}

\section{BORDER AND REGIONAL INTEGRATION IN HEALTH: ACCESS TO THE HEALTH SYSTEMS OF POSADAS - ENCARNACIÓN}

\author{
Liliana Elizabeth WOLHEIN ${ }^{2}$ \\ Nora Margarita JACQUIER ${ }^{3}$ \\ Etelbina Raquel OSORIO ${ }^{4}$ \\ Ruth Noemí MARTÍNEZ ${ }^{5}$
}

\begin{abstract}
Resumo: O ano de 2020 nos surpreendeu com um evento extraordinário de Saúde Pública em nível global, que travou áreas de fronteira integracionistas em quase todo o planeta. Estudos sobre o acesso à saúde nas fronteiras na perspectiva da cidadania social e da saúde como direito humano, em uma área específica do Mercosul, mostram que as assimetrias existentes entre os sistemas de saúde de cada um. dos países membros, que influenciam o limitado avanço das políticas integracionistas na matéria. Algumas experiências europeias e do Mercosul de cooperação fronteiriça na área da saúde permitemnos reconhecer que a cidadania social e o acesso universal à saúde podem materializar-se considerando a densidade de cada um dos espaços - tempos particulares, como a fronteira PosadasEncarnação.
\end{abstract}

Palavras-Chave: Acesso universal à saúde; Cidadania social; Fronteira; COVID-19.

Abstract: The year 2020 surprised us by an extraordinary Public Health event at a global level, which put a lock on integrationist border areas in almost the entire planet. Studies on access to health care at the borders from a perspective of social citizenship and health as a human right, in a specific area of Mercosur, show that the existing asymmetries between the health systems of each of the member countries, which influence the limited progress of integrationist policies in the matter. Some European and Mercosur experiences of border cooperation in the health field allow us to recognize that social citizenship and universal access to health can be materialized considering the density of each of the spaces - particular times, such as the Posadas-Encarnación border.

Keywords: Universal access to health; Social citizenship; Frontier; COVID-19.

Resumen: El año 2020 nos sorprendió un acontecimiento de Salud Pública extraordinario a nivel global, puso un candado a los espacios fronterizas integracionistas en casi todo el planeta. Los estudios sobre el acceso a la atención de la salud en las fronteras desde una perspectiva de ciudadanía social y de la salud como un derecho humano, en un espacio específico del Mercosur, dan cuenta que las

\footnotetext{
${ }^{1}$ Este texto sintetiza una parte de los resultados de una investigación realizada en el marco de una consultoria 2017 - 2018. Coordinada por la Prof Dra. Vera Nogueira- UFSC -UCPel denominado "Cidadania em zona de fronteira - O caso do MERCOSUL" financiado por el Instituto Social del Mercosur y por outra la resultados de la investigación $16 \mathrm{Q} 633$.

${ }^{2}$ wolheinlili@gmail.com; Escuela de Enfermería. Facultad de Ciencias Exactas Químicas y Naturales. SECIPUNaM. Especialista en Educación Superior. Ciencias y Servicios de Salud

${ }^{3}$ norajacquier@gmail.com - Escuela de Enfermería. Facultad de Ciencias Exactas Químicas y Naturales. SECIPUNaM. Magíster en Salud Pública. Ciencias y Servicios de Salud.

${ }^{4}$ eraqueloso@gmail.com; Escuela de Enfermería. Facultad de Ciencias Exactas Químicas y Naturales. SECIPUNaM. Licenciada en Enfermería. Ciencias y Servicios de Salud.

${ }^{5}$ ruthnoemimartinez@gmail.com Escuela de Enfermería. Facultad de Ciencias Exactas Químicas y Naturales. SECIP- UNaM. Especialista en Educación Superior. Ciencias y Servicios de Salud.
} 
asimetrías existentes entre los sistemas de salud de cada uno de los países miembros, que influyen en el escaso avance de políticas integracionistas en la materia. Algunas experiencias europeas y mercosureñas de cooperación fronteriza en el campo sanitario permiten reconocer que la ciudadanía social y acceso universal a la salud pueden ser materializadas considerando la densidad de cada uno de los espacios -tiempos particulares, como es la frontera Posadas-Encarnación.

Palabras Claves: Acceso universal de Salud; Ciudadanía social; Frontera; COVID-19.

\section{Introducción}

El año 2020 nos sorprendió un acontecimiento de Salud Pública extraordinario a nivel global, que pone en suspenso concepciones fronterizas integracionistas en casi todo el planeta. Este trabajo surge de la ponencia (Wolhein et al, 2020) presentada en el "V Seminario Internacional de los Espacios de Frontera (GEOFRONTERA) Territorialidades y Sujetos transfronterizos" celebrado en octubre de 2019, en la Facultad de Humanidades y Ciencias Sociales de la Universidad Nacional de Misiones-, y en el marco del eje "Fronteras, integración y estados nacionales". En esa ocasión, nos habíamos propuesto reflexionar sobre el acceso a la atención de la salud en las fronteras desde una perspectiva de ciudadanía social y de la salud como un derecho humano, en un espacio específico del Mercosur. Pero, el contexto de pandemia global por el Covid-19 declarado por el director del organismo rector de la salud mundial, el doctor Tedros Ghebreyesus (Organización Mundial de la Salud, 2020a) nos interpela a ampliar la mirada, debido a que se ha generado un panorama de gran incertidumbre por la propagación del virus que desencadena enérgicos mecanismos de control social en las zonas fronterizas.

Nos preguntábamos y nos preguntamos si las asimetrías existentes entre cada uno de los países miembros del MERCOSUR y los Estados Asociados del bloque influyen en el escaso avance de políticas integracionistas en materia sanitaria y en este marco, particularmente las asimetrías de los sistemas nacionales de salud entre las ciudades vecinas Posadas y Encarnación. Nuestro interés se centra en mostrar las características y brechas de las desigualdades que cada país tiene en su interior, como son las diferencias entre los sistemas nacionales de salud. Uno de los ejes fundamentales del Plan Estratégico de Acción Social (PEAS) del MERCOSUR es "Universalizar la salud Pública" sin embargo no se evidencia un avance en los Programas en materia Sanitaria para el acceso de los ciudadanos del MERCOSUR. La Cartilla de ciudadanía del bloque describe determinados programas en el campo educativo, la seguridad social, las normas sanitarias para intercambios de felinos y 
caninos domésticos, asimismo, da cuenta de la ausencia de propuestas que incorporen a la Salud Humana (MERCOSUR, 2015).

A esta problemática se suma la falta de articulación del desarrollo social y el crecimiento económico, reflejadas en la disponibilidad de infraestructura y acceso a bienes y servicios que tienen los ciudadanos, derivando en una construcción de ciudadanía social propia de cada país. El presente trabajo tiene por objetivos analizar las convergencias y divergencias para la atención de extranjeros en las secretarías de salud de las ciudades fronterizas Posadas, Argentina y Encarnación, Paraguay. Asimismo, reconocer propuestas de integración regional en salud en dichas ciudades fronterizas. Para ello fue necesario discutir algunas aproximaciones posibles a los conceptos de frontera y políticas públicas de integración en salud, ciudadanía social y algunas experiencias de integración fronteriza en el campo sanitario.

El estudio es de tipo cualitativo, utilizando la técnica de recolección de datos entrevista en profundidad. Los sujetos de estudio fueron gestores sanitarios de las secretarías de salud de Posadas -Encarnación. El escenario fue recortado a la zona de frontera Posadas Encarnación, donde la confluencia geopolítica de ambos países adquiere regional para el Mercosur debido a la relevante interacción social y económica. La técnica empleada para la recolección de datos fueron entrevistas, en las que los investigadores de campo fuimos debidamente identificados con un kit conteniendo la presentación personal y el consentimiento informado libre y esclarecido. Las entrevistas en profundidad contenían preguntas que buscaban indagar sobre la existencia de dispositivos jurídicos y administrativos (protocolo, legislaciones, acuerdos, etcétera) de los países de Argentina y Paraguay vinculada a la salud de frontera.

Asimismo, se plantean interrogantes referidos el acceso a la salud de los no Nacionales, sobre los principales problemas en la atención a los servicios de salud prestados y requisitos para la atención. La sistematización del material obtenido consistió en tareas de transcripción de las entrevistas grabadas y la carga de datos en la matriz analítica y cuadros de los servicios prestados según las entrevistas. Las asimetrías regionales (Instituto Social del MERCOSUR, 2018a) analizadas constituirían un punto de partida para la búsqueda de propuestas integracionista viables, antes que se desencadenara la pandemia del COVID-19. 


\section{Aproximaciones a la atención de la salud en la frontera}

Tiempos de pandemia, tiempos de un nuevo coronavirus, tiempos de repliegue de las fronteras que demarcan los estados-nación. El proceso de cuidar la salud y atender la enfermedad de la población residente en el área de fronteras a partir de una perspectiva regional es compleja y tiene diversos planos de análisis. Uno de los planos, es que los sistemas de salud nacionales y las políticas sanitarias, como toda política pública, están impregnados tanto por las ideologías, como por los valores de cada uno de los países involucrados (Jacquier, 2014) y en este sentido, las estructuras e interacciones del sistema de salud materializan diferentes proyectos, según las ideologías y valores que los actores sociales de los grupos hegemónicos tienen sobre proceso salud-enfermedad-atención (Menéndez, 2015). Asimismo, los estados modernos se instituyeron sobre principios de libertad e igualdad promulgados en la Declaración Universal de los Derechos Humanos (Organización de Naciones Unidas, 1948), en el que se inscribe el Derecho a la Salud. Pero, para el antropólogo indio, Partha Chatterjee (2011) la ciudadanía universal es uno de los grandes relatos de la modernidad; en la que la ciudadanía es una forma de vida limitada a pocas personas.

Otro aspecto a tener en cuenta constituye la noción imperante de frontera en cada uno de los países limítrofes. Las nociones de frontera y salud son retomadas de enfoques de la geografía crítica, antropología política y médica crítica. Bauman (1998) propone repensar al espacio de frontera como un objeto dinámico socialmente construido. Consideramos que la geografía crítica ayuda a comprender las diferencias de algunos términos que entran en juego en este texto: línea, zona y región de frontera. La zona de frontera es una franja territorial más amplia que la "línea fronteriza" creada por un estado-nación hacia el interior de dicho límite con diferentes criterios.

El Decreto 887 del Poder Ejecutivo Nacional Argentino establece que las zonas de frontera son "zonas de seguridad" para "la defensa nacional" y comprenden "una faja a lo largo de la frontera terrestre y marítima". Más adelante, aclara que "será variable y el Poder Ejecutivo lo fijará según la situación, población, recursos, e intereses" con un "máximo de $150 \mathrm{~km}$ en la frontera terrestre".

Geógrafos como Harvey, Santos y Soja consideran que la realidad política y económica determina la estructuración espacial, en la cual, "los cambios antrópicos producidos sobre el espacio (espacio socialmente construido) son condicionados por las 
necesidades del ser humano, de forma tal que la naturaleza, ya humanizada, refleja las relaciones desiguales entre las clases sociales”. (BARRERA, 2009.p.13).

Para Abinzano (2004) las fronteras antropológicas suponen la posibilidad de áreas de mezcla cultural entre diferentes sistemas de contacto y estas relaciones se producen traspasando líneas fronterizas. Este mismo autor sostiene que la región de frontera es "una noción antropológica que implica la presencia del factor espacial" que se enfoca principalmente en el complejo sistema de interacciones de las praxis" y no en el establecimiento de sus límites, pues los fenómenos que ocurren en esa región tienen una peculiaridad especial.

Estas ideas llevan a rever que tanto las fronteras internacionales son líneas imaginarias delineadas por el hombre, así como la salud es otro constructo teórico social-cultural-histórico y políticamente determinado pero que son dinámicas y que la utilidad se encuentra en la dimensión de la praxis, es decir del espacio vivido. (Jacquier et al, 2013)

Según Paasi (en Benedetti, 2014) el límite es una línea divisoria que separa el "nosotros" de los "otro/s". Cuando las fronteras son vistas como un ámbito complejo de confluencia de diferentes tipos de prácticas estatales y nativas, es una perspectiva que trasciende a la tradicional considerándola como una línea que separa y divide tanto países, como personas. Ambos enfoques coexisten, una visión integracionista concibe a las fronteras como espacios porosos; pudiendo aplicarse la perspectiva de Lefebvre (en Barrera Lobaton, 2009) que categoriza al espacio como un espacio "concebido" o como un espacio "vivido". Las categorías espaciales ayudan a repensar posibilidades para la colaboración y la solidaridad en salud transnacional.

\section{La zona de frontera Posadas-Encarnación}

Las fronteras geopolíticas son dinámicas como la vida misma. En el seminario señalábamos la importancia del cambio de perspectiva, concebidas no como barreras ni como pasos abiertos, más bien entendidas como flujos diferenciados, desiguales en su tratamiento en el momento del cruce de los límites. A partir de esta comprensión “...se puede entender los efectos que tiene esta clasificación sobre la sociedad en la frontera, así como los efectos de las fronteras sobre la sociedad" (Heyman, 2011, p: 82). Las regiones fronterizas son espacios complejos, en ellas coexisten elementos de integración y de conflictos. Al mismo tiempo que 
se discute y aborda esta temática se debe tener en cuenta que en esos lugares las políticas sociales y económicas implementadas en un estado afectan directamente a la población vecina.

La noción del espacio cartesiana-positivista es una visión tradicional que lo caracteriza como algo dado, rígido y mensurable. (Harvey, 2006). En el espacio cartesiano de la doble Frontera confluyen dos estados- nación, Argentina y Paraguay. Tanto Posadas, Argentina y Encarnación, Paraguay son consideradas por algunos autores como ciudades gemelas o ciudades espejo.

Los países de Argentina y Paraguay están situados en América del Sur. Paraguay está dividido por el río Paraguay en dos regiones bien diferenciadas, la Región Occidental o Chaco, que cuenta con tres departamentos; y la Región Oriental con 14 departamentos. La superficie total del país es de $406.752 \mathrm{Km} 2$.

La pertenencia a diferentes naciones también refleja diferentes datos estructurales en cuanto al índice de desarrollo humano -IDH-, el Producto Bruto Interno -PBI- per cápita En el año 2017, la población del Paraguay fue de 6.811.297 habitantes según Banco Mundial. El Ingreso Nacional Bruto -INB - per cápita método Atlas (anteriormente PBI per cápita) fue de 3.920 (año 2017). De acuerdo con el Informe de Desarrollo Humano de 2016, el Índice de desarrollo Humano -IDH- de Paraguay fue de 0.69 y ocupa el puesto número 110 a nivel mundial y el puesto 19 en Latinoamérica, categorizada con IDH "medio".

La Argentina es un estado federal descentralizado, dividido en 23 provincias y la Ciudad Autónoma de Buenos Aires instituida como la capital del país. En términos geográficos la Argentina posee una superficie total de 3.745.247 kilómetros cuadrados. Integra el MERCOSUR y es el segundo mayor país del bloque.

Su área total de frontera es de 9.376 kilómetros limitando con cinco países: Bolivia, Paraguay, Brasil, Chile y Uruguay. El Ingreso Nacional Bruto -INB- per cápita en Argentina es de 13.040 en el año 2017. De acuerdo con el Informe de Desarrollo Humano de 2016, el IDH de Argentina es de 0,827, ocupando la posición número 45 entre los países en un ranking con 188 países. De acuerdo con el ranking mundial es uno de los dos países del cono sur que se encuentra entre los 50 primeros, que figuran en la lista de desarrollo humano caracterizado como "muy alto" y en segundo lugar en Latinoamérica después de Chile.

No obstante Naciones Unidas advierte que la desigualdad persiste en el continente. Al observar IDH al incluir la desigualdad, la región registra una pérdida total de 23,4\% y, por 
ejemplo, Argentina retrocede en esa clasificación 6 puestos. En el ranking del IDH de América Latina y el Caribe: Argentina (45), y Paraguay (110).

Siguiendo con la descripción a continuación se mencionan algunos datos de las ciudades elegidas para el desarrollo de este trabajo Posadas-Encarnación. Uno de los ocho pasos fronterizos legales más característico de la Provincia de Misiones es el Paso Internacional San Roque González de Santa Cruz, donde se ubica el Centro de Frontera Posadas- Encarnación. De un lado se encuentra la ciudad de Posadas ubicada en la provincia de Misiones, Argentina la cual está en el extremo noreste del país casi totalmente rodeada por ríos (Paraná, Iguazú y Uruguay) tiene una extensa frontera internacional; al oeste con Paraguay, al norte y este con Brasil. La población de Posadas según el Instituto de Estadísticas y Censos tiene una proyección para el año 2016 es de 293.578 habitantes.

Del otro lado del río nos encontramos con la ciudad de Encarnación capital del departamento de Itapúa que ancla sus raíces a orillas del río Paraná. Es la tercera ciudad más importante del Paraguay, detrás de Asunción y de Ciudad del Este. Alberga a una población predominantemente urbana de 127.527 (2016) habitantes.

\section{Sistemas de Salud en Posadas y en Encarnación}

Como resultados de las convergencias y divergencias para la atención de extranjeros en las secretarías de salud de las ciudades fronterizas Posadas-Encarnación, se analizó los Sistemas de Salud- SS- desde el plano estructural dentro de los sistemas oficiales de salud de cada uno de los países. Estos sistemas presentan diferencias en relación con la forma de organización y oferta de los bienes, acciones y servicios según cada país.

La alta fragmentación es un rasgo que caracteriza históricamente al sistema de salud argentino, además de la segmentación comprende un sector público, uno privado y el de las Obras Sociales. También, el sistema paraguayo es del S.S en público y privado. El subsector público está representado por el Ministerio de Salud Pública y Bienestar Social, el Instituto de Previsión Social, la Sanidad Militar, la Sanidad Policial, la Universidad Nacional de Asunción y los servicios asistenciales de las empresas descentralizadas Itaipú y Yacyretá. Esta fragmentación contribuye a diferentes modos de exclusión social en salud para la población. Aragüés y Oroz, (2017, p: 25) sostienen que se observa una estrecha relación entre nivel 
EM

QUESTÃO

V.13 N. $04 \bullet 2020$

pág. 132-147

socioeconómico de la población, lugar de residencia, tipo de cobertura y acceso institucional a los servicios y, en definitiva, posibilidades de salud.

Los sistemas de salud configurados en desiguales subsistemas tienen diferentes tipos de convenios para la financiación, afiliación y provisión a segmentos de población de acuerdo con su nivel de ingresos y por su condición social. Este tipo de arreglo institucional consolida y profundiza la desigualdad entre los diferentes grupos poblacionales en el acceso a la salud y es un factor de exclusión en salud porque es un escenario de alta segmentación, los más pobres, desinformados y menos poderosos se quedan fuera del sistema. (MICHAUX, 2004)

Además, se han analizado los criterios de acceso y documentos exigidos en el Sistema de salud. En Posadas se puede decir que en el primer nivel tanto municipal-provincial la documentación requerida es la acreditación de la identidad -DNI-, que no es excluyente para extranjeros, garantizando de igual manera la atención registrando DNI en trámite. Encarnación, no cuenta con criterios de acceso, la gestora refiere: "Se atiende a todos por igual, a los argentinos, paraguayos y brasileños en los hospitales públicos" y agrega que no hay demanda de Posadas [argentinos] a los sistemas de salud en Encarnación, solamente en la parte privada para implantes bucales...”. La prestación de servicios de salud pública difiere entre ambos países para los no nacionales.

Los servicios sanitarios en Posadas incluyen atención médica y de especialidades gratuitas, guardias médicas y de enfermería, acceso a programas de salud provinciales y nacionales; el segundo y tercer nivel están arancelados. En Encarnación incluye internación, cirugía, carece de servicios de alta complejidad como terapia intensiva y diálisis; de programas para patologías crónicas sólo acceden personas con radicación paraguaya.

Las políticas de integración Regional se enmarcan en procesos integracionista y regionalista, según Zeraoui (2017) el primero busca reforzar las relaciones transfronterizas y facilitar los intercambios culturales y sociales, además de aspectos exclusivamente económicos. El segundo centra las propuestas regionalistas en las afinidades entre los estados, por ejemplo, en el Cono sur se aglutinó en MERCOSUR, que incluyó además a los estados Asociados al Bloque (Chile, Perú, Colombia, Ecuador y también Bolivia).

Desde el Bloque se han propuesto diversas políticas regionales que buscan disminuir las asimetrías y mejorar los estándares mínimos de integración social y política (Mondelli, 2017). Dentro de estas políticas se impulsó en el año 2010 un plan de acción para conformar un Estatuto de Ciudadanía del MERCOSUR (EC) para el año 2021. Según Mondelli (2017) 
EM

QUESTÃO

V.13 N. $04 \diamond 2020$

pág. 132-147

"el EC promueve ampliar la libertad de movimiento y la integración social equitativa de las poblaciones de la región" (p.36).

Esto se plasma en la Cartilla de la ciudadanía del MERCOSUR donde existe una compilación de normas relacionadas con el ciudadano y la ciudadana del MERCOSUR. En este sitio es posible reconocer distintas áreas donde son presentadas estas normativas, por ejemplo, la circulación de personas y bienes asociada a la búsqueda de medios y procedimientos que faciliten la libre circulación de mercaderías, de factores productivos y personas. En el área de educación se presentan normativas vinculadas a la Gratuidad de visas para estudiantes y docentes de los Estados Partes del MERCOSUR, formación docente de posgrado, reconocimientos de títulos para el ejercicio de actividades académicas entre otros aspectos.

Sin embargo, en el área de aspectos sanitarios y de salud algunas de las normativas vigentes se enfocan a las normas sanitarias para el intercambio en el MERCOSUR de caninos y felinos domésticos, procedimientos mínimos de inspección sanitaria en embarcaciones que navegan por los estados Parte del MERCOSUR, Control y salida de estupefacientes y sustancias psicotrópicas dejando de lado normativas específicas para la salud humana.

En el contexto de la Unión de Naciones Suramericanas -UNASUR ${ }^{[1]}$ - la salud es un principio, en cuanto a cooperación e integración regional, que prioriza el desarrollo de las políticas públicas y modos superadores en materia de diplomacia sanitaria, principalmente respecto al Banco de Precios de Medicamentos, el Mapeo de capacidades productivas de medicamentos y el Plan Integral para el cáncer de cuello de útero (rigazzi et.al,.2018, p.2)

Una de las escasas políticas de integración en materia de salud de las personas, es la de unificación de criterios para el registro de la atención sanitaria de la población materno infantil aprobada el año 2005, por la Resolución del Grupo Mercosur Común (GMC) $\mathrm{N}^{\circ}$ 04/05 que establece la Información Básica Común que debe contener la Libreta De Salud Del Niño/A de los Estados Parte del MERCOSUR. Esta norma fundamenta, "la necesidad de contar con información básica común en un instrumento transportable que permite la continuidad de atención de los niños que se desplacen de un Estado Parte a otro”. El artículo 2 hace referencia a que la edad definida para usar la Libreta que fue determinada por cada uno de los países miembros, así como podrá agregar otros requisitos según sus propias normativas nacionales o locales. La aplicación de las reglamentaciones del GMC se dan a través de cada uno de los organismos nacionales de la cartera, siendo en Argentina, el Ministerio de Salud y 
Ambiente; en el Brasil, el Ministerio da Saúde; en Paraguay, el Ministerio de Salud Pública y Bienestar Social y en el Uruguay, el Ministerio de Salud Pública.

En cuanto a las propuestas de integración regional ambas ciudades carecen de proyectos de integración sanitaria (se realizan acciones sanitarias para problemas de salud vectoriales) y de mecanismos formales de referencia-contra referencia. Los gestores manifiestan la intencionalidad de integración transfronteriza en perspectiva de derechos humanos, pero hasta el momento no está acompañada de acciones concretas, saben que existen acuerdos para la atención de enfermos paraguayos en el hospital, pero no está vigente. Incluso, los niños argentinos que viven en el Paraguay tienen dificultades en acceder a la atención médica en los servicios de mayor complejidad, sobre todo cuando deben ser derivados.

La búsqueda de atención sanitaria de paraguayos en la ciudad de Posadas se debe a que la oferta del servicio de salud es excelente comparativamente con los suyos, cuenta con servicios de mayor nivel de complejidad y provisión de medicamentos. Los gestores de ambas ciudades refieren que intentan disminuir las barreras de acceso a los sistemas locales de salud de manera informal dado que la salud de frontera es un asunto de competencia nacional. Un obstáculo importante para la toma de decisiones racionales es la falta de una base de datos y de convenios locales sobre el uso de servicios a nivel regional (Posadas-Encarnación) por parte de los no nacionales.

Existen acuerdos en instancias supranacionales como el MERCOSUR que lleva tiempo en materializarse. En tanto, en otras se de aplicación inmediata como es particularmente, el acuerdo realizado entre la Cámara de Comercio Internacional (ICC) y la Organización Mundial de la Salud para la colaboración estrecha entre los sectores públicos y privados en que en la coyuntura de la pandemia se amplia el sentido dado a los espacios fronterizos que permiten la libre circulación de productos del mercado para la atención sanitaria como sucede actualmente en el Puente Posadas-Encarnación:

Los gobiernos deben comprometerse a habilitar todos los recursos necesarios para combatir la COVID-19 con la mínima demora posible y garantizar el funcionamiento efectivo y eficiente de las cadenas transfronterizas de suministro de productos médicos y otros bienes esenciales (Organización Mundial de la Salud, 2020b). 


\section{Experiencias de cooperación en otros espacios fronterizos}

Pérez y Nogueira (2009) describen experiencias positivas de cooperación transnacional, dos en el ámbito europeo: Extremadura - Alentejo, España y Cataluña, EspañaPuigcerdá, Francia y una en el MERCOSUR. La primera iniciativa tendió a incrementar la cohesión económica y social a través de políticas regionales y estructurales, con el apoyo de capitales europeos, creados con el fin de cooperar en fronteras transnacionales o interregionales, para desarrollar comunidades de manera armoniosa, equitativa y sostenible.

Estas acciones parten de los gestores locales, que con sus propios recursos están en condiciones de identificar las dificultades comunes y sin solución, alentando asimismo que la demanda continua de servicios de salud en las fronteras permanezca bajo la responsabilidad de los países involucrados. En el campo administrativo los servicios y los gobiernos establecen mecanismos de registro de prestación de servicios y reglas de compensación; pactos formales de garantía de acceso a grupos específicos de pacientes, como ejemplo, la atención prenatal y los partos, siendo los pagos debidos acordados entre los gobiernos nacionales (Pérez y Nogueira, 2009:55).

La segunda experiencia Puigcerdá-Cataluña, en la región de los Pirineos, un área turística y agropecuaria. Hasta fines de la Segunda Guerra Mundial, el hospital español atendía a pobladores sin distinción de su nacionalidad. En la actualidad conforma una red sanitaria eficaz, con alta resolutividad de casos de media y alta complejidad.

Desde el año 2000, a través de un acuerdo, las parturientes son derivadas al Hospital de Puigcerdà. Los nacimientos son registrados como si fueran en territorio francés y el pago realizado a través de un convenio establecido hace seis años con la Agencia Regional de Hospitalización y Seguro Médico, con la anuencia de la Prefectura Municipal de Perpignan. Posteriormente al inicio de la atención en Puigcerdà las autoridades francesas y españolas visitaron el Hospital y resolvieron construir otro con división de responsabilidades entre Francia y España. (...) A principios de 2008, se iniciaron los protocolos comunes sometidos a sociedades científicas de los dos lados de la frontera. Se inició un debate acerca de una Red Sanitaria Transfronteriza, con la participación de profesionales de ambos países (Pérez y Nogueira, 2009:55).

Otra experiencia, pertenece al ámbito del Mercosur, en la Triple Frontera entre Brasil, Argentina y Paraguay, las mismas autoras sostienen que cada uno los sistemas de salud generan desiguales niveles de protección sanitaria, particularmente, para la población paraguaya que busca atención en los países vecinos. Una de las mayores demandas es la de la atención de los partos. En respuesta a esta demanda, en la ciudad de Foz do Iguaçu se creó 
una institución exclusiva para la atender partos de ciudadanas paraguayas, en las proximidades del puente internacional.

Según Da Silva (2014) ese Centro específico también fue creado para evitar que las mujeres embarazadas brasileñas que viven en Paraguay utilicen directamente el servicio de atención al parto en Brasil, porque frecuentemente concurren sin antecedentes de sus controles reproductivos y prenatales. Al igual que ocurre con mujeres paraguayas que residen en Encarnación y que como estrategia cruzan a la ciudad de Posadas, en período avanzado del trabajo de parto para evitar el rechazo de la atención médica. En Brasil, el lugar de residencia influye fuertemente en el acceso a los servicios de salud, así como en la clasificación positiva de la atención por parte del usuario, aumentando la posibilidad de que busque o no la unidad de salud más cercana a su domicilio.

Por último, una de las experiencias más exitosas es la de frontera mercosureña, Santana do Livramento, Brasil y Rivera, Uruguay que se caracteriza por ser una región fronteriza que presenta importantes avances documentados y legitimados para garantizar el derecho a la salud, sin distinción de nacionalidad para a los residentes. El Sistema Único de Salud exige -SUS- la presentación de la tarjeta del SUS para acceder a la atención sanitaria. Un acuerdo informal entre ambas localidades dispone que la primera atención de urgencia o emergencia debe darse. La implementación de una tarjeta fronteriza garantiza la atención en el SUS, sin distinguir a los ciudadanos uruguayos. también acceden a Servicios Especializados para las enfermedades de transmisión sexual, como el HIV/SIDA, la sífilis, la hepatitis y a programas de reducción de daños para los usuarios de drogas. Otra de las particularidades de esta región fronteriza es la contratación de médicos ginecólogos para la Santa Casa de Santana do Livramento, por falta de postulantes brasileños. Del lado uruguayo, sucede lo mismo frente a carencia de profesionales especialistas en determinada área, "se não tem a especialidade, o médico vem, opera e o hospital lhe paga", não necessita de autorização judicial (Instituto Social del MERCOSUR, 2018:99).

Finalmente, la experiencia europea solidaria en el contexto de pandemia, en la que Alemania brinda servicios sanitarios a miembros de la comunidad europea para la atención de personas afectadas de COVID-19, es un hecho que revela como se ponen en juego tanto valores como principios en los procesos de cooperación transfornteriza (Infobae, 2020). Estas prácticas ad-hoc son excepcionales y vienen incluso antes de la Segunda Guerra Mundial de procesos de integración, generalmente, se caracterizan por la particularidad de los gestores locales que poseen voluntad política y fuerte compromiso con la vida de las personas que 
viven en la región. Son experiencias que muchas veces se inscriben acuerdos informales, en la "paradiplomacia" en la búsqueda de alternativas para el bien común. (Instituto Social del MERCOSUR, 2018).

\section{Consideraciones Finales}

Como conclusión podemos decir que debemos hablar de fronteras, cada una tiene sus particularidades. Existen fronteras en las que han encontrado modos sui géneris de materializar prácticas para ampliar la cobertura universal de salud y cristalizar la ciudadanía social en salud, incluso en la actual situación de pandemia global. El estudio muestra una frontera estructuralmente desigual y heterogénea en los sistemas de salud de la Frontera Posadas-Encarnación que influyen en la respuesta que se da a los sujetos sociales según su ciudadanía.

El análisis de los aspectos que favorecen tanto las diferencias como las convergencias entre los sistemas de salud de los dos estados-nación permite repensar el espacio de la doble Frontera como espacio de posibilidad de colaboración y solidaridad en salud y no como espacio para la defensa. Tal como lo expone los gestores del sector salud de los países quienes plantean que existe predisposición para la integración de tareas en materia de Salud Pública y plantear acciones conjuntas. Sin embargo, no se concretan en las acciones y servicios de salud destinados a los usuarios de servicios de salud extranjeros.

La distinta especificidad en la política sanitaria y la organización del sistema entre los países fronterizos dificultan el trato de las desigualdades de acceso y atención. Por lo tanto, es distinta la gestión de las necesidades de salud en la frontera en los países que presentan un sistema sanitario centralizado en la planificación y gestión, que en otros países donde la descentralización de la gestión y organización de los recursos ha desarrollado las intervenciones en el territorio a partir de sus especificidades, incluso en el caso de que ambos presenten similares objetivos universales de salud.

Ante lo expuesto la propuesta es buscar una posible complementación para que la frontera sea permeable como oportunidad para ampliación del derecho a la salud que requiere de trabajo interdisciplinario, articulado entre diferentes sistemas de salud.

Es así que como principal desafío se podría pensar en una planificación y gestión de la Salud que considere las particularidades de las regiones fronterizas buscando corregir las 
distorsiones de la distribución de los recursos de salud observadas, considerando las desigualdades regionales de condiciones de vida, situación de salud y accesibilidad. Buscando así extender la atención a la totalidad de sus poblaciones, mejorando la distribución espacial de los servicios (cobertura y accesibilidad geográfica), y aumentando la capacidad instalada, inclusive para la atención de media y alta complejidad.

\section{Referências}

ABINZANO, Roberto. Antropología de los Procesos Transfronterizos: conocer y actuar en la región de fronteras. Cuadernos de la Frontera, Año I. Posadas: Secretaría de Investigación y Postgrado. FHyCS, UNaM, 2004.

ARAGÜÉS Y OROZ, Valentín. Documento sobre rectoría, Funciones Esenciales de Salud Pública, federalismo y sistema de salud argentino. En: Artaza Barrios, O. FESP: su implementación en Argentina y desafíos hacia la salud universal: experiencia federal. 1a ed. Buenos Aires: OPS, 2017, p. 19-43.

ARGENTINA. DECRETO $\mathrm{N}^{\circ}$ 887- ANEXO I. Límite de la zona de frontera para el desarrollo de la Ley 15.575 y de Zonas de Seguridad de frontera - Decreto Ley 15.385/44 y Ley 12.913. 1994.

BARRERA LOBATÓN, Susana. Reflexiones sobre Sistemas de Información Geográfica Participativos (sigp) y cartografía social. Cuadernos de Geografía: Revista Colombiana de Geografía, Bogotá, n. 18, p. 9-23, may. 2009.

BAUMAN, Z. Urban Space Wars: On destructive order and creative chaos. Space and Culture, Estados Unidos, v. 2, p. 109-123, dic.1998.

BENEDETTI, Alejandro. Espacios fronterizos del sur sudamericano: Propuesta de un modelo conceptual para su estudio. Estudios fronterizos, Mexicali, v. 15, n. 29, p. 11-47, ene/jun 2014.

CHATTERJEE, Partha. La política de los gobernados. Revista Colombiana de Antropología, Bogotá, v. 47, n. 2, p. 199-231, jul-dic. 2011.

HARVEY, David. A reinvenção da geografia: uma entrevista com os editores da New Left Review. En: HARVEY, David. A produção capitalista do Espaço. São Paulo: Annablume, 2006, p. 15-40.

HEYMAN, Josiah. Cuatro temas en los estudios de las fronteras contemporáneas. En: Ribas Mateos Natalia. El Río Bravo Mediterráneo. Barcelona: Ediciones Bellaterra, 2011, p. 81-98. Infobae. Cómo es por dentro el avión hospital que Alemania envió a Italia para recoger a enfermos de coronavirus. Buenos Aires, 2020. Recuperado en: https://www.infobae.com/america/mundo/2020/03/30/al-interior-del-imponente-avion-militarque-alemania-envio-a-italia-para-recoger-a-enfermos-de-coronavirus/ 
INSTITUTO SOCIAL DO MERCOSUL. Cidadania Social no MERCOSUL. Acesso a serviços sociais em regiões de fronteira, Assunção, 2018.

JACQUIER, Nora. Salud Pública y Comunitaria. En Jacquier [et al.] Introducción a la enfermería comunitaria una contribución a la salud colectiva. Posadas: Creativa, 2014, p. 2949.

JACQUIER, Nora y CENTENO, Jorge. ¿Servicios de Salud Interfronterizos, una Utopía? Revista Sociedade em Debate. Pelotas, v. 19, n. 2, p. 110-131. 2013.

MENENDEZ, Eduardo. De sujetos, saberes y estructuras. Introducción al enfoque relacional en el estudio de la salud colectiva. Buenos Aires: Lugar Editorial, 2015.

MERCOSUR. Resolución GMC No 04/96 y Resolución GMC No 17/15. Buenos Aires, 2015. Recuperado en: http://www.cartillaciudadania.mercosur.int/oldAssets/uploads/RES_017-

2015_ES_Caninos\%20y\%20Felinos\%20Domesticos\%20-\%20no\%20vigente.pdf

MERCOSUR/GMC/RES. N ${ }^{\circ}$ 04/05. Información Básica Común Para La Libreta De Salud Del Niño/A. Recuperado en: https://www.mercosur.int/documentos-y-normativa/resoluciones/

MICHAUX, Jacqueline. Hacia un sistema intercultural de salud en Bolivia. De la tolerancia a la necesidad sentida. En: Fernández, G. (coord.). Salud e interculturalidad en América latina. Perspectivas antropológicas. Quito: Abya Yala, 2004, p. 107-129.

MONDELLI, Marcelo. Elementos para profundizar la agenda del MERCOSUR ciudadano. Revista MERCOSUR de políticas sociales. Asunción, v. 2, p. 34-59, nov. 2018.

NACIONES UNIDAS PARA EL DESARROLLO -PNUD. Informe sobre Desarrollo Humano 2016 Desarrollo humano para todas las personas. 2016. Recuperado en: http://www.undp.org/content/dam/undp/library/corporate/HDR/HDR2016/HDR_2016_report _spanish_web.pd

NACIONES UNIDAS-CEPAL. Agenda 2030 y los Objetivos de Desarrollo Sostenible. Una Oportunidad para América Latina y el Caribe. 2017. Recuperado en http://repositorio.cepal.org/bitstream/handle/11362/40155/10/S1700334_es.pdf

Organización de Naciones Unidas. Resolución 217 A (III) de la Asamblea General de las Naciones Unidas. 1948.

ORGANIZACIÓN MUNDIAL DE LA SALUD. Alocución de apertura del Director General de la OMS en la rueda de prensa sobre la COVID-19. 2020a. Recuperado en: https://www.who.int/es/dg/speeches/detail/who-director-general-s-opening-remarks-at-themedia-briefing-on-covid-19---11-march-2020

ORGANIZACIÓN MUNDIAL DE LA SALUD. Declaración conjunta de la ICC y la OMS: Un llamamiento a la acción sin precedentes dirigido al sector privado para hacer frente a la COVID-19-2020b. Recuperado en: https://www.who.int/es/news-room/detail/16-03-2020-iccwho-joint-statement-an-unprecedented-private-sector-call-to-action-to-tackle-covid-19 
PEREZ JIMENEZ, Roser y NOGUEIRA, Vera Maria Ribeiro. La construcción de los derechos sociales y los sistemas sanitarios: los desafíos de las fronteras. Revista katálysis, Florianópolis, v. 12, n. 1, p. 50-58, ene-jun. 2009.

PROVINCIA DE MISIONES. Proyección de población según municipio. Período 2010 2020. Recuperado en: https://ipecmisiones.org/wp-content/uploads/2019/04/IPEC-MisionesEstimaciónde-la-Poblacion-de-Misiones-por-municipio-2010-2020.pdf

RIGGIROZZI, Pía.; HERRERO, María y TUSSIE, Diana. Viraje Político y la Agenda Regional En Salud. Cuadernos Del Pensamiento Crítico Latinoamericano. Consejo Latinoamericano de Ciencias Sociales. Buenos Aires: CLACSO, 2018.

SILVA, Maria Geusina. O Pacto Pela Gestão E A Saude Na Fronteira: Condições E Relações De Trabalho Dos Assistentes Social Em Debate. Porto Alegre: Faculdade De Serviço Social, Pontifícia Universidade Católica do Rio Grande do Sul, UCePel, 2012. Número de páginas 218. (Tese Doutorado).

SVAMPA, Maristella. Reflexiones para un mundo post-coronavirus. En: SVAMPA, M; CRAGNOLINI, M. RIBEIRO S, AIZEN S, LÓPEZ M P, RODRÍGUEZ ALZUETA E, et al. La Fiebre. Editorial Aislamiento Social Preventivo y Obligatorio: 2020, p. 17-37.

ZERAOUI, Zidane. Más allá de la frontera. Los estudios fronterizos en la actualidad. En Repensar las fronteras, la integración regional y el territorio/Ed. ACOSTA, Willy. $1^{\circ}$ ed., Heredia, Costa Rica: CLACSO, IDESPO, Universidad Nacional de Costa Rica, 2017. 